situated in the posterior part of the first (upper) temporosphenoidal convolution (and perhaps in the adjacent part of the second temporo-sphenoidal convolution) and that in the great majority of normal individuals this centre is only active (or only sufficiently active independently to carry on the auditory speech function) on one (the left) side of the brain. Consequently, the absence of any lasting word deafness in Edgren's case is easily explained.

Further, if it be granted that the lower centre for hearing (i.e., for ordinary sounds) and the auditory tone centre are situated in the first or upper temporo-sphenoidal convolution, the lesions which were found in this (Edgren's) case would seem to show (1) that the anterior end of the first temporo-sphenoidal convolution is the lower auditory centre for ordinary sounds; and (2) that the middle part of the temporo-sphenoidal convolution is the auditory centre for musical sounds (the auditory tone centre). In Edgren's case the anterior two-thirds of the first (upper) temporosphenoidal convolution on the left side and the posterior half of the first (upper) temporo-sphenoidal convolution on the right side were destroyed. If one were to divide the first (upper) temporo-sphenoidal convolution into three parts and to locate the auditory centre for ordinary sounds in the anterior part, the auditory centre for musical sounds in the middle part, and the auditory centre for speech "sounds in the posterior part it would be easy to give an intelligible explanation of the auditory symptoms which were present in this case. On the left side the posterior third of the first left temporo-sphenoidal convolution (the auditory speech centre) remained intact, consequently there was no word deafness. On the right side the anterior part of the first temporo-sphenoidal convolution, which I have suggested may perhaps be the lower auditory centre for ordinary sounds, remained intact and since this centre is active on both sides of the brain the patient was still able to hear and understand ordinary sounds. The middle part of both first temporo-sphenoidal convolutions which I have suggested is perhaps the auditory tone centre, was completely destroyed on the left side and partly destroyed on the right side of the brain. This would explain the persistence of the "tone deafness," granting - as I think is probably the casethat the auditory tone centre is, in most normal individuals, active on both sides of the brain. But it must be remembered that such a division of the first (upper) temporo-sphenoidal convolution (the auditory centre as a whole) is purely theoretical. It takes it for granted that the centres for ordinary hearing, for musical sounds, and for speech sounds are all situated in, and confined to, the first or upper temporo-sphenoidal convolution. But, as I have already pointed out, A. W. Campbell's researches seem to show that the lower centre for hearing is situated in the transverse temporal gyri of Heschl; and the cases of word deafness which have been examined post mortem seem to show that the auditory speech centre occupies more than the posterior third of the first (upper) temporo-sphenoidal convolution.

In the present position of our knowledge it is impossible to make an explicit statement as to the exact position of the auditory note centre; but for the reasons given above $I$ am disposed to think that it is probably situated in front of the anditory speech centre, in or near the middle part of the first (upper) temporo-sphenoidal convolution or the adjacent portion of the transverse temporal gyri of Heschl, and that in most normal individuals it is probably active on both sides of the brain. Consequently, if this localisation is correct, complete and permanent "tone deafness" will, in most normal individuals, only result from a bilateral lesion which destroys the auditory note centre (the middle part of the upper temporo-sphenoidal convolution or perhaps the adjacent portion of the transverse gyri of Heschl) in each hemisphere of the brain.

One would, a priori, expect that the auditory centre for musical sounds would, like the auditory centre for ordinary sounds, be active on both sides of the brain, and the fact that in many cases of auditory aphasia (word deafness) in which the first (upper) left temporo sphenoidal convolution is extensively destroyed there is no permanent or persisting "tone deafness" seems to give support to this view. It must, however, be remembered that in other cases of word deafness in which the same part of the brain is destroyed the presence of "tone deafness" has been noted, at all events as a temporary condition.

It is unnecessary to say that the musical faculty varie very greatly in different individuals and it is quite possible that in some persons-i.e., in those who inherit a strong musical faculty - the auditory tone centre is active on both sides of the brain, whereas in other persons-i.e., those who have little or no natural musical faculty but in whom a certain degree of musical proficiency is acquired as the result of training and cultivation-the auditory tone centre is active only on one (the left) side of the brain. Further, one may suppose that in the case of highly cultivated and trained musicians who have been taught to read music at sight and to write music the auditory tone centre which was probably in them originally active in both hemispheres of the brain may have come to be chiefly active (located) in the left hemisphere, since there is reason to suppose that the visual tone centre (like the visual speech centre) is in such cases only or chiefly active in one (the left) hemisphere. ${ }^{16}$

The examination of cases of aphasia would seem to show that the emissive or motor centre for the vocal expression of musical sounds is active on both sides of the brain; at all events this would appear to be the fact in those cases of motor-vocal aphasia in which the patient can sing words which he is unable to pronounce in the form of ordinary speech.

\section{PRIMARY PNEUMOCOCCAL ARTHRITIS.}

By W. PASTEUR, M.D. Lond, F.R.C.P. LOND., PHYSICIAN TO THE MIDDLESEX HOSPITAL; AND

L. COURTAULD, M.A., M.B., B.C., D.P.H. Cantab., ASSISTANT IN THE BACTERIOLUGICAE TABORATORY, MII)DLESEX HOSPITAL.

PNeUmococcal affections of joints are well recognised and many instances of the condition have been placed on record. In the great majority of these, however, the joint affection has been secondary, in point of time, to some other pneumococcal lesion of the body, such as pneumonia, empyema, or otitis ; or has arisen in the course of a general infection (with endocarditis, meningitis, \&c.); whilst the cases in which an arthritis of this nature has occurred as an isolated phenomenon, in the absence of any other signs of infection, so as to merit the name "primary," are few in number.

Frequenoy: (a) In adults.-The condition would appear to be very uncommon. Among 56 recorded cases of arthritis due to pneumococcus only two were of primary origin. These 56 cases have been derived from the following sources. Leroux, in his excellent monograph on the subject, collected 26 instances of pneumococcal affections of joints; 23 of these were associated with pneumonia, Of the three remaining, one was strictly primary-that of Widal and Lesné, of which an abstract is given below. In the second case there was found after death a purulent pericarditis, of pueumococcal origin, which was apparently of an earlier date than the arthritis. In the last case it is doubtful whether the joint affection preceded or followed the cerebral lesion. The patient was suffering from an acute inflammation of the ankle-joint and from a right-sided hemiplegia, which was shown after death to be due to a pneumococcal meningitis. No history of the illness could be obtained.

Cave has appended three additional cases to his summary of Leroux's paper, each of them consequent on pneumonia. Raw has had under his own care eight patients suffering from this condition, in each case as a sequel of pneumonia. Herrick has collected 13 examples, but one only, that of Allen and Lull, which is referred to later, was primary. Quite recently Secretan and Wrangham have described six more cases with a history of recent pneumonia in every one. Last year Fornaca published another example of the primary form of pneumococcal arthritis, which, with the case recently in the Middlesex Hospital, brings the total up to four Without in any way claiming to be exhaustive, this analysis serves to emphasise the rarity of the primary form of the disease.

(b) In children.-In early life, affections of joints due to pneumococcus, whether primary or secondary, seem to be

1" In a paper on "Crossed"Aphasia (The Lancet, June 3rd, 189? p 1473). I have directed attention to the influence which the acquirement of the act of reading and writing, perhaps, has in locating the active or driving speech centres (auditory and motor vocal) in the left hemisphere of the brain. The same reasoning applies to the musical faculty. 
of more common occurrence. Dadgeon and Branson state that in their experience " pneumococcus practically monopolises the etiology of infective arthritis during infancy and about up to the end of the first hemi-decade." Often there is also present some inflammation of the middle ear and the great importance of this association must be insisted on as providing a probable explanation of the origin of the arthritis. Otitis media is one of the most frequent results of pneumococcal activity during the early years of life. Netter examined 46 consecutive cases of pneumococcal infection in children and found amongst them 29 instances of otitis media. It is a reasonable supposition that at times the inflammatory process in the middle ear may be so trivial in its clinical manifestations as to be entirely overlooked and yet of sufficient severity from a pathological standpoint as to afford the organism an entry into the blood stream and enable it to set up what is apparently a primary arthritis.

Dudgeon and Branson have recorded five cases of suppurative arthritis (pneamococcal) in children. In two of these there was an accompanying otitis media ; in one a history of preceding pneumonia; whilst in two the joint affection was the only discoverable lesion. Herzog has published an account of 28 collected cases occurring in children under the age of two years. In four of these no other evidence of infection could be found. He lays particular stress on the importance of disease of the middle ear as a precursor of this condition; he also quotes several other lesions which have been suggested as possibly holding a similar relationship, such as conjunctivitis (Cagnani), follicular stomatitis (Ockmar), and catarrhal angina of the oral cavity (Bichat and Coffert).

Combining these two sets of observations, it is found that out of 38 cases of pneumococcal arthritis in infants and young children, six were primary, a marked contrast to the corresponding figure for adults. But it is not improbable that the frequency of this condition at all ages is greater than these figures would seem to indicate. For when an arthritis of this nature arises quite apart from any signs of infection elsewhere and the patient recovers without operation, it is more than probable that the nature of the pathogenic organism will remain undiscovered.

Pathology. - The exact mode of origin in these cases is not fully understood. It is evident that the channel of infection is the blood stream, and the access of any micro-organism to the blood stream presumably implies some injury to the smaller vessels at the point of attack leading to stasis and possibly to thrombosis. Through the devitalised vessel walls the organisms make their way or small fragments of clot containing bacteria may become detached and infection is in this way disseminated throughout the circulation.

As regards pneumococcus, when this organism determines an arthritis, subsequently to some well-marked lesion elsewhere, such as pneumonia, meningitis, or otitis, the site of entry is evident. Pn the absence, however, of any such antecedent one must assume that a slight injury of a mucous membrane or of the skin has provided the means of entry. It is in this sense that we have applied the term "primary" to the joint lesion-viz., as implying that the real initial lesion-the spot where the organism has gained access to the circulation-has been so slight as to give no indications of its situation. There is reason to believe that this usually occurs in the middle ear, mouth, naso-pharynx, or tonsils. Diplococcus pneumoniæe is almost constantly present in the saliva and is therefore at hand to seize any favourable oppor tunity of effecting an entrance into the circulation. Moreover, it has come to be recognised within recent years that this parasite has an important influence on the causation of sore-throat, with or without membranous exudation, very similar to that met with in diphtheria. If a very slight attack of this nature were to pass entirely unrecognised both by patient and medical attendant any ensuing complication would be likely to be classed as primary.

It is therefore evident that there are no grounds for drawing a hard-and-fast line between primary and secondary pneumococcal arthritis. For whether the initial injury be slight or severe, the minute pathological processes which enable the organisms to invade the blood stream are very similar, any difference that exists being one of degree only. At the same time there is a distinction which may be made between the two varieties and which is not without importance in prognosis.

Where the arthritis is primary and localised there is no reason to assume any extensive or lasting infection of the blood. It is reasonable to suppose that by some means or other a batch of pneumococci, possibly a very small one, set free in the circulation has alighted on one of the structures of a joint the protective mechanism of which is at the moment a little below the average, whether owing to injury or some other cause, and has given rise to the inflammation. It is probable that the initial lesion-the site of entry-so trivial that it has escaped detection, will have healed before any considerable number of organisms have made their way into the blood stream. A similar explanation may be applicable to cases of gonorrhceal and certain other forms of infective arthritis. Later, no doubt, the infection may become generalised and the condition pass into one of true septicæmia, the seat of distribution of the organisms being in this event the inflamed joint and not the small initial lesion.

To turn now to a case of secondary arthritis, arising, say, during an attack of pneumonia. The conditions met with here form an almost exact antithesis to those just described. The initial lesion is a gross one, the injury to the lung tissue is severe, and its powers of resistance are almost annibilated for the time being. Moreover, there is an enormous supply of organisms at hand to continue the invasion of the blood stream and these of a degree of virulence much enhanced by their successful struggle with the tissue cells. This distinction, if true, suggests a difference in severity between primary and secondary arthritis of pneumococcal origin, and the hypothesis seems to be borne out by the actual facts. For, after making due allowance for the small number of cases, the recovery of three out of the four patients with primary arthritis described below is in marked contrast with the death-rate of about 65 per cent. for the cases of secondary arthritis.

There remains a group of cases which, from a pathological standpoint, is very puzzling-namely, those in which the symptoms of the joint affection appear some days before the lung trouble. Leroux mentions two cases of this kind in boys, aged respectively five and eight years. In the firs of these the artbritis preceded the pneumonia by three days, and in the second by seven days; the former died and the latter recovered. Raw describes the case of a man, aged 5 ? years, in whom the arthritis preceded the pneumonia by two days; the patient recovered. There are two possible explanations of this unusual sequence of events. Either the arthritis is the primary event and the pneumonia secondary or, where the interval is short, the infection of the joint takes place during the period of incubation of the pneumonia. Owing to this uncertainty of origin we have not classed these cases as primary.

It is not quite clear why diplococcus pneumoniæ should at times show a selective preference for the structures of a joint. Not infrequently there is a history of some previous injury which has apparently determined the localisation of the infection. For instance, Billings describes the case of a man who, shortly before the crisis of pneumonia, became delirious and jumped out of a second-storey window, injuring his left shoulder and right knee. Some days after the crisis both joints became acutely inflamed and distended with pus containing pneumococci. Again, in the case of a newly born child, mentioned below, the joint attacked had been previously injured durins a difficult labour. Further, the previous injury may be of pathological origin. Thus, rheumatism and gout appear to render the joints less able to resist bacterial invasion. In this connexion Widal draws attention to the importance of "eauses internes," such as deficient innervation, in affording what he calls a "point d'appel" to organisms.

Experiments on animals throw some light on the question. Ausset inoculated mice and rabbits subcutaneously with cultures of pneumococcus after applying some mechanical injury ("traumatisme aseptique") to the joints. In seven out of nine animals there ensued a purulent arthritis. Züber obtained a similar result by the simultaneous injection of terebinth into the joint cavity and of pneumococci beneath the skin, whilst Kasparek has shown that section of the sciatic nerve favours the localisation of any subsequently inoculated organisms in the bones and joints of the enervated limb. But the most interesting series of experiments are those of Bezançon and Griffon who were able to produce an inflammation of the joints of rabbits either by injecting virulent cultures into partially immunised animals or by using cultures for inoculation attenuated by age. In these circumstances they found that the animals did not, as is usually the case, die from a generalised infection within from 24 to 48 hours but developed localised lesions, 
especially of the joints, appearing some considerable time after inoculation. They were unable to recover the organism from the tissues after death but found that the serum of the animals had an active agglutinative reaction on pneumococci and also a protective influence against experimental inoculation. Besançon and Grifton regard these results as bearing out the general pathological law that "microbes of attenuated virulence readily attack the various serous structures, especially those of the joints." This line of argument implies that a primary arthritis of pneumococcal origin is to be explained either by weakness of attack or by unwonted resistance on the part of the patient, but this is a matter of conjecture. Nevertheless these experiments serve to emphasise a point of extreme weight in the consideration of the phenomena of bacterial pathogenesis-namely, that these phenomena depend on two factors, the activity and vitality of the invading organism and the defensive powers of the subject attacked, two factors inseparately associated and, as it were, reciprocal; and in order to arrive at any true understanding of the real significance of pathogenesis it is necessary to consider the one with the other.

The degree of immunity or, conversely, of susceptibility in any given instance which finds expression in the severity or variety of the pathological processes that occur, represents the balance struck between these two opposing forces ; and inasmuch as both the one and the other are of uncertain stability and liable to variation, so also the susceptibility will be similarly inconstant, and may even become converted into an immunity - that is to say, an organism usually pathogenic may lose its toxic properties.

Illustrative cases. - There follows a short account of four cases of primary pneumococcal arthritis. The first of these refers to a patient recently under the care of one of us (W. P.) in the Middlesex Hospital. The other three have been collected from the literature. A description of a fifth case is appended, which, though not strictly primary, is for many reasons of interest.

CASE 1. - A young man, aged 23 years, landed in England on Sept. 24th in good health and was admitted to the Middlesex Hospital on the following day suffering from an acute inflammation of the right knee-joint. His family history was excellent and he had always enjoyed good health. Four months previously he had a fall from his bicycle, slightly bruising both knees, but was quite recovered in a few days. He had never had gonorrhoea. On the morning after his arrival in London, whilst out walking, he noticed a slight "pricking" sensation in his right knee. This soon gave place to pain which compelled him to lie up on the same afternoon. 'The onset of the illness was not attended by any sore-throat nor by any feeling of general malaise. On admission the temperature was $102^{\circ} \mathrm{F}$, and the face was a little flushed. The right knee-joint was visibly distended by fluid, very tender, and acutely painful. No other joint was affected. The tongue was somewhat furred; the throat was healthy. The thoracic organs were normal and there were at no period of his illness any symptoms of lung disease. The urine was acid, clear, and did not contain flakes. Hot fomentations were applied to the knee and sodium salicylate was prescribed. For the first four days the knee continued to increase in size though the pain gradually became more bearable. On Sept. 29th the temperature had gradually fallen to normal but rose again the same evening and continued to oscillate between normal and $102^{\circ}$ until after operation on Oct. 12th when the fever quickly subsided. On Oct. 9th the knee-joint was aspirated and two or three ounces of thin greenish-yellow sero-pus were withdrawn, with marked relief to the patient. A very few capsulated diplococci, exactly resembling pneumococci, were found in the fluid and it was decided to drain the joint. Mr. Andrew Clark operated on Oct. 12th, making a longitudinal incision about two inches long on either side of the joint. A large quantity of greenish sero-pus was evacuated, the joint was thoroughly washed out with warm boric solution, a drainage tube was inserted, and a cyanide gauze dressing was applied. The patient suffered severe pain in the joint the night after operation and was given morphine. The joint was irrigated daily and a considerable quantity of pus came away for the first few days. The discharge of pus had ceased on Oct. 24th and from this date convalescence was rapid. He was sent to the Clacton Convalescent Home on Nov. 8th and returned a few weeks later restored to health and with a perfectly sound joint.

Bacteriologioal examination.-The examination of the fluid withdrawn from the knee by an exploring needle on Oct. 9th gave no very definite results. It is true that in cover-glass specimens, stained by Löfller's blue and by Gram's method, there could be seen with the microscope a few scattered diplococci resembling diplococcus rneumonix both in shape and in staining reactions, but the inoculation of artificial media (three agar and three broth tubes) produced no growth. Moreover, the injection of some of the fluid into the peritoneal cavity of a mouse caused no harmful effect to the animal. However, the microscopical appearances were alone sufficient to justify a strong suspicion that the arthritis was due to the pneumococcus. The case seemed, therefore, to be one of exceptional interest and accordingly an extended series of observations were made with material obtained at the time of the operation. As soon as the joint cavity had been opened some of the blood-stained sero-pus which emerged was collected in a sterilised syringe and utilised as follows :-

1. Cover-glass preparations were made and stained by Löffler's blue and Gram's methods. These showed a small number of diplococci the appearance of which was exactly typical of the pneumococcus. Though few in number they were rather more numerous than those seen in the first specimen of sero-pus. The capsules were very well marked.

2. From the pus were inoculated 37 tubes of various media (agar-agar, glucose-agar, horse serum, gelatin, peptone broth, glucose broth, lactose, and litmus broth); also two Erlenmeyer flasks, each containing 75 cubic centimetres of peptone broth, and one Erlenmeyer flask containing 75 cubic centimetres of milk. The flasks were used because this method had often proved successful in attempting to isolate an organism from fluids in which it is present only in very small numbers when inoculation of tubes in the ordinary way has given a negative result. In this particular case no growth occurred on any of the tubes and one of the flasks also remained sterile. In the other flask of broth a slight general turbidity was seen after incubation at $37^{\circ} \mathrm{C}$. for 48 hours. The medium was examined microscopically after 64 hours and was found to contain diplococci retaining Gram's stain, and subcultures from this broth on various media corresponded in every way with those of the pneumococcus. After 64 hours the milk in the third flask showed signs of commencing coagulation. On microscopical examination there were seen a few diplococci but these were quite outnumbered by very many large Gram-staining bacilli. The latter were obviously a contamination, such as is frequently present in milk tubes presumed to be sterile but which have not been tested before inoculation by incubation for 12 hours at $37^{\circ} \mathrm{C}$.

3. Some of the pus was injected into the peritoneal cavity of a rabbit and of a mouse; both these animals remained alive and well. Finally, two cubic centimetres of the broth culture mentioned above was injected into a mouse with a similarly negative result.

Comments - Owing to the failure of the animal experiments the final test of the identity of the organism was lacking, nevertheless there is no reason to doubt that the arthritis was caused by pneumococcus. We base the diagnosis of primary arthritis on the complete absence of sore-throat or general malaise at the time of onset and do not consider that the slight bruising of the knees four months ago invalidates this view of the case. One is naturally tempted to connect the mildness of the illness with the harmless effects of animal inoculation, but such an astonishing discrepancy is sometimes found to exist between the toxicity of an organism towards man and towards animals that the inference would not in our opinion be justified.

Case 2 (Widal and Lesné, quoted by Leroux, 1899).-The patient was a man, aged 68 years, in the enjoyment of perfect health. He gave no history of previous illness, except that he had been subject to a slight degree of rheumatism in the smaller joints of the hands and feet. One morning, whilst at work apparently in his usual health, he was suddenly seized with severe chills, headache, and general feeling of illness. He was feverish that evening and slightly delirious at night. Next morning he complained of diffuse pains in the left arm which on the following day became localised in the left sterno-clavicular joint and the back of the left hand. On admission to hospital on the eighth day of illness it was found that the left sterno-clavicular joint was swollen to the size of a pigeon's egg and was very tender. On the back of the left hand there was an acutely tender swelling over the third and fourth metacarpal bones. 
No fluctuation could be detected in either place. The lungs were quite healthy and no other evidence of disease could be found. The temperature on admission was $102 \cdot 2^{\circ} \mathrm{F}$.; on the following day it fell to normal and did not rise again above this level. The swelling at the back of the hand gradually disappeared during the next few days; that of the sterno-clavicular joint, however, slowly increased in size until fluctuation was felt, and this in spite of a complete absence of general symptoms. A sterilised pipette was introduced into the joint and a few drops of pus were withdrawn. This was found to contain a number of capsulated diplococci, which were proved to be identical with diplococcus pneumoniæ by means of cultures on artificial media and the inoculation of animals. After this the pain and swelling slowly diminished. For eight days they remained entirely absent, and then, without apparent reason, reappeared. Some pus was again withdrawn and examined with exactly similar results. Once more the local symptoms slowly subsided and the patient left the hospital completely cured six weeks after admission.

Comments.-This case presents several points of interest : the sudden onset, resembling pneumonia ; the short duration of the constitutional symptoms; the curious recrudescence of the inflammatory process in the sterno-clavicular joint without accompanying rise of temperature. Leroux regards the rheumatic changes in the joints as having been an important factor in determining the situation of the infective process.

CASE 3 (Allen and Lall, 1902).-The patient was a woman, aged 40 years, single, who sought admission to hospital on Jan. 24th on account of pain and swelling in the left knee of two days' standing. During the four days preceding admission she had suffered from "cramps in the abdomen." The patient was deeply offended at being asked if she had had gonorrhcea and absolutely refused to give any account of her illness. The general condition was good although the temperature was $101.4^{\circ} \mathrm{F}$. On the following day the joint was distended with fluid and some thick, yellow pus was withdrawn with an aspirator. It was decided at once to lay open and thoroughly to cleanse the joint. On Jan. 27th the general condition was worse with delirium and high fever $\left(103^{\circ}\right)$. The leg was amputated above the knee. The stitches and gauze-drainage wicks were removed on Feb. 3rd when two ounces of sero-pus escaped. The tissues about the wound looked very unhealthy. On the following day the patient's condition was very serious with rigors, delirium, and high fever, the temperature reaching $108^{\circ}$ just before death, which occurred that evening.

Bacteriological examination.-The pus from the kneejoint was found to contain typical capsulated diplococci and cultures made from it yielded a growth characteristic of the pneumococcus. No experiments on animals were made. Cultures made from the blood during life remained sterile. Post-mortem examination revealed no other traces of pneumococcal infection.

Comments.-On account of the negative result of the bacterial examination of the blood Allen and Lull regard the fatal ending of this case as due to severe toxæmia, the organisms themselves being confined to the joint but disseminating their poisonous products throughout the body. If this supposition be correct the case is one of exceptional interest. It is, however, well known that the cultivation of organisms from the blood is a matter of some uncertainty and the failure of one attempt to do so cannot be considered as a proof of their absence unless confirmed by repetitions of the attempt.

CASE 4 (Luigi Fornaca, 1905).-The patient was a woman, aged 28 years, married, in the seventh month of pregnancy. On Nov. 15th while in apparent good health she was suddenly seized with severe headache, shivering, and a sense of general illness. Four days later she complained of intense pain in the right elbow and a few hours later in the right knee. She was admitted to hospital on Nov. 19th, the eighth day of illness. The right knee-joint was swollen to thrice its natural size, the skin covering the joint was tense but not reddened, and fluctuation could not be obtained; there were acute pain and tenderness on manipulation. The pain and swelling in the elbow had completely disappeared on admission and the joint appeared to be normal. The pharynx and tonsils were somewhat inflamed and the latter were slightly swollen. On the skin over the right knee-joint were two small pustules, and there was a similar one on the left knee. There was a crop of herpetic vesicles round the mouth. The patient's general condition was good. For the first ten days the temperature ranged between $101^{\circ} 5^{\circ}$ and
103. During this time the headache and rigors continued with spasmodic attacks of pain in the affected joint. After this the temperature assumed a remittent type, finally falling to normal on Dec. 14th. On the second day after admission an exploratory puncture was made into the right knee-joint and some sero-purulent fluid was withdrawn. Aspiration was repeated several times, the withdrawal of fluid from the joint affording much relief. The swelling of the joint remained for ten days after admission and then slowly sub. sided. The patient was discharged cured on Dec. 19th.

Bacteriological exumination. - A full examination was made on the second day of the fluid withdrawn from the joint and of the contents of the pustules. Each of these was found microscopically to contain numerous capsulated diplococc the appearance of which was exactly typical of diplococcus pneumoniz. Cultures made from these two sources on to agar produced a pure growth of characteristic dew-drop colonies. Some of the pus from the joint was injected into several mice and rabbits, both subcutaneous and intraperi toneal injection being employed. None of the animals died, though some of the mice were obviously ill. But it was found that the virulence of the organisms contained in this pus could be raised by two successive passages through animals to a point at which they were able to produce a fatal result. On the third day after admission a bacteriological examination was made of (1) blood arawn from the median vein; (2) milk squeezed from the breasts; and (3) urine. In each case direct microscopical examination gave a negative result but cultures made in peptone broth yielded a typical growth. These bacteriological examinations were repeated on the fifth and tenth days after admission with a similarly positive result but on the fourteenth day the result was negative.

Comments.-The author refers to the slight inflammation of the tonsils as being the probable initial lesion in this case but he says that there was no exudation or membrane in that region of which a bacteriological examination could be made. Peculiar interest attaches to the wide dissemination of the organisms which is in surprising contrast to the slight severity of the illness. We have failed to find any other record of the isolation of the pneumococcus from the milk and the urine.

The following case, though not strictly primary, is mentioned here on account of the close resemblance which it bears to the laboratory experiments of Ausset, described above.

CASE 5. (Nattan-Larrier, 1905).-This concerns a male infant, born on Sept. 5th, after a long and tedious labour, the presentation being left occipito-anterior. The forceps was not used. At birth the child was found to have a harelip, otherwise his condition was satisfactory. The mothe was quite healthy. Three days after birth the edges of the hare-lip were freshened and united by sutures. The wound in the lip did not heal well and on Sept. 12th there was a slight serous oozing from it, which on the 15 th was distinctly purulent. The child's general condition did not, however, suffer until some days later (the 20th) when there were loss of appetite and vomiting. On the 24th the mother noticed that the boy cried when laid on his right side or when the right shoulder was touched; the genera condition was unsatisfactory and the child was losing weight. Two days later he was gravely ill and was admitted to hospital. The shoulder was wollen to the size of a mandarin orange and the skin over the joint was red and odematous; deep fluctuation could be felt. The spleen was enlarged. The temperature was $100 \cdot 4^{\circ} \mathrm{F}$. Death occurred on the next day. At the post-mortem examination 50 grammes of thick yellow pus were found in the cavity of the shoulder-joint. This pus contained in large numbers an organism which satisfied all of Koch's postulates as to it identity with the pneumococcus. The discharge from the lip was not examined until after death when it also was found to contain numbers of pneumococci. No trace of pneumo coccal infection was found in any other part of the body.

Comments. - Nattan-Larrier, with reason, supposes that the right shoulder suffered some injury during labour. Such an event is not uncommon and might in this case explain the localisation of the infective process. If this be so, the injury would exactly correspond to the aseptic traumatism employed by Ausset, whilst the entry of the organisms through the wound in the lip would be closely analogous to ubcutaneous inoculation.

Conclusions. - 1. Primary pneumococcal arthritis is appar. ently of rare occurrence in adults. 2. In children it is less uncommon. 3. The path of infection is the blood stream, 
to which the organisms gain access through some slight and annoticed area of injury. This is in all probability situated in the mouth, naso-pharynx, or middle ear and the relative frequency of this form of arthritis in infants and young children may be connected with their susceptibility to inflammatory conditions of these regions. 4. The infection is usually localised at first in one joint and may remain so ; on the other hand, it may become generalised. 5. The death-rate in the primary form compares very favourably with that of secondary pneumococcal arthritis. This may be accounted for by the usual restriction of infection in the former, whereas the latter generally implies a more or less pronounced septicæmia. 6. It is probable that the records available do not convey an accurate idea of the frequency of this form of arthritis, as many slight cases, which recover without surgical interference, must escape bacteriological examination.

Bibliography.-D. P. Allen and C. Lull : Annals of Surgery, October, 1901. Bezançon and Grifton: Société de Biologie, July, 1899. E. J' Cave: The Lancer, Jan. 12th, 1901, p. 82. 1. S. Dindgeon and Fornaca : Giornale dell'Accademia di Medicina di Torino, March, 1905. J. B. Herrick : American Journal of the Medical Sciences, July, 1902. Hans Herzog: Jahrbuch fur Kinderheilkunde, April, 1906. Iéon Leroux : Les Arthrites ì Pneumocoques, Paris, 1899. Nattan-Larrier: Archives Générales de Médecine de Paris, 1, 1905. W Pasteur: December, 1901. W. B. Secretan and W. Wrangham: Brit. Med. Jour., April, 1906.

Middlesex Hospital, W.

\section{A CASE OF CONGENITAL HYPERTROPHY OF THE PYLORUS.}

\section{BY J. W. ROB, M.D. CANTAB.}

THE rarity of this disease and the absence of agreement amongst the chief authorities as to the best treatment of it make the publication of every case advisable. The following is a brief summary of the main symptoms as expressed by most of the writers on the subject. From 80 to 85 per cent. of the cases are in males. The family history is un. important. The symptoms do not appear until the baby is about a month old and then the primary symptom is persistent, intractable, copious, forcible vomiting which is uninfluenced by diet. No apparent cause for the vomiting exists. The characteristic feature of the vomiting is its forcibleness. It is not frequent and there is no bile in the vomit. There are progressive loss of weight, visible gastric peristalsis, a tumour in the pyloric region, dilatation of the stomach, constipation as a rule, and a normal or subnormal temperature.

The patient in my case was born on Oct. 25th, 1905, and was a fine, healthy boy. At first he was fed at the breast and seemed to be doing well but on Nor. 16th, as he did not seem to be gaining weight he was fed on a mixture of cow's milk (one part) and barley water (two parts), two ounces every two hours. On the $19 \mathrm{th}$, as there was no improvement he was given the same amount of a mixture of milk (two parts) and barley water (three parts) with one drachm of cream in each ounce. There was no improvement, however, on this food and the child became very constipated. On the 20 th he vomited for the first time at 9 A.M., about an hour after a feed. The amount of the vomit was about four ounces. It consisted only of undigested food. The vomiting came on suddenly without obvious cause and was very forcible, the contents of the stomach being vigorously pumped up in big gushes. About 6 P.M. on the same day a second vomit of about three ounces occurred. The average capacity of the stomach of a baby of a month old being about from two and a half ounces to three ounces the stomach in this case was already dilated at least a third. The temperature was normal; there did not seem to be any abdominal pain and the tongue was clean. On the 21st the food was changed to equal parts of milk, lime-water, and water, but the baby vomited again twice at the same hours, about the same amount, and in the same way.

Dr. Robert Hutchison saw the baby on Nov. 22nd. The question of hypertrophy of the pylorus was raised but dismissed because no gastric peristalsis could be seen. This was no doubt due to the fact that the baby vomited shortly before examination. Vomiting occurred on this day the same as previously. Wasting was now obvious to the eye. The epigastrium was prominent. The temperature was $97 \cdot 8^{\circ} \mathrm{F}$. The food was changed to a mixture of peptonised milk (one part) and water (two parts) with one orachm of milk sugar in each feed. No benefit resulted from this. Vomiting continued as before. The temperature remained subnormal. Constipation was still troublesome. On the 24th one-sixth of a grain of mercury with chalk was given three times a day but with no result. In the evening of this day, as vomiting had already taken place three times, no food but only hot water in doses of one drachm every half hour was given for six hours. The vomiting, however, continued. On the 25th the food was changed to Allen and Hanburys No. 1 and two grains of carbonate of bismuth were given every two hours. The child was by this time very wasted indeed. The face was white and shrivelled, the eyes were sunken, and the lips were blue. The skin was hanging in loose folds on the arms and legs. The temperature was $97^{\circ}$. The epigastrium was full. Gastric peristalsis was clearly seen. Dr. Hutchison saw the baby again in the evening. The child was fed just before examination and gastric peristalsis was well marked. No definite pyloric tumour could be felt. A diagnosis of hypertrophy of the pylorus was made and the line of treatment adopted was that of washing out the stomach every day and feeding on peptonised milk (one part) and water (two parts) in feeds of half an ounce every hour. The question of operation was discussed on this occasion and also on Dec. 9 th.

The accompanying chart tells the history from this date. The baby was weighed after the stomach had been washed out. He was undressed completely and wrapped in a shawl which was weighed alone afterwards. The temperature continued subnormal until Dec. $18 \mathrm{th}$, when it rose to normal, where it has since remained. Constipation was a difficulty at first. On Nov. 27th one-sixth of a grain of calomel was given every hour until the bowels acted. This was repeated on several occasions until Dec. 11th. On that day in addition to the milk one drachm of somatose was dissolved in ten drachms of cold water and two drachms of brandy were added; one drachm of this mixture was given after each feed. Also two drachms of maltine were mixed with eight drachms of water and one drachm of the mixture was added to each feed. The effect of the somatose on the constipation was excellent. The bowels, as a rule, after this acted regularly. Once or twice the motions were dark in colour and slimy but this only lasted a day or two. In February the somatose was discontinued but as constipation returned it was begun again. The weight steadily decreased until Dec. 11th in spite of the vomiting having become less. The daily records during this period were as follows: 6 pounds on Nov. 25th; 6 pounds 1 ounce on Nov. 26th; 6 pounds 3 ounces on Nov. 27 th; 6 pounds 2 ounces on Nov. 28th; 5 pounds 15 ounces from Nov. 29 th to Dec. 2nd; 5 pounds 12 ounces on Dec. 3rd; 5 pounds 9 ounces on Dec. 6 th ; and 5 pounds 7 ounces on Dec. 9 th and 11th.

It is difficult to describe the appearance of the baby at the beginning of December. Nobody really believed that he would live. He was wasted to an extreme degree and was so feeble that it was a matter of surprise that he was in existence at all. The dilatation of the stomach continued for a long time. At the end of February the stomach held six ounces easily, whereas the normal capacity of the organ at the age of four months is only four ounces. Peristalsis was well marked until the middle of January when it disappeared. A pyloric tumour was felt on Dec. 4th distinctly, not very distinctly about a fortnight later, and not at all about the middle of January. The quantity of food taken is shown on the chart. At first half-ounce feeds were given every hour. On Nov. 29th this was increased to six drachms every hour, on Dec. 1st to one ounce every hour and a half, on the 3 rd to 12 drachms every two hours, and on the 5 th to two ounces every two hours, and on Jan. Ist to three ounces every two and a half hours. At first, though the baby seemed hungry, he did not take his food well. A few mouthfuls satisfied him and the remainder of the feed had usually to be given with a spoon. However, this passed off about the end of December and he has since taken his food well from a bottle.

On Dec. 5th as the child was only vomiting once a day and the amount was not so large as formerly nor the vomiting so forcible it was decided to wash out the stomach on alternate days and to give one-eightieth of a minim of tincture of opium 20 minutes before each feed. This was 